IAPs? And what is Sickle's actual function in vivo? More insight into the genetic interaction between sickle and other cell-death-regulating genes in Drosophila is required to address these questions.

Two proteins that seem to be functional homologues of Reaperlike proteins have recently been identified in mammals. These proteins Smac/DIABLO and Omi/HtrA2 also bear the conserved amino-terminal motif. However, their expression pattern and subcellular localization seem to be different from those of the Drosophila Reaper-like proteins, so further work is needed to establish whether they are true homologues.

Valerie Ferrier, Associate Editor, Nature Cell Biology

(2) References and links ORIGINAL RESEARCH PAPERS Srinivasa, M. et al. sickle, a novel Drosophila death gene in the reaper/hid/grim region, encodes an IAP-inhibitory protein Curr Biol. 12, 125-130 (2002) | Wing, J. P. et al. Drosophila sickle is a novelgimuaper P. et al. Drosophila sickle is a novel grim-reaper cell death activator. Cur. Biol. 12, 131-135 (2002) | Christich, A. et al. The damageresponsive Drosophila gene sickle encodes a novel IAP binding protein similar to but distinct from reaper, grim, and hid. Curr. Biol. 12. 137-140 (2002)

\section{WEB SITES}

Emad Alnemri's laboratory:

http://www.kcc.tju.edu/staff/alnemri/

John Nambu's laboratory:

http://www.bio.umass.

edu/biology/faculty/inambu.phtm

John Abrams' laboratory:

http://www.swmed.edu/home_pages/cellbio/dw/ abrams\%20people.html

enlarging speckles.

This indicated that Sox proteins might be directly involved in splicing, which the authors then showed to be the case - splicing was inhibited and spliceosomal complexes failed to form in SOX6-depleted nuclear extracts. However, adding back recombinant SOX6, SRY or SOX9 restored splicing in a dosedependent manner, confirming a role in pre-mRNA splicing.

As Sox proteins have unusual DNA-binding properties, there is every possibility that they can bind and bend RNA too. By doing so, they could then facilitate the rearrangements that occur during splicing.

Katrin Buss (2) References and links ORIGINAL RESEARCH PAPER Ohe, K., Lalli, E. \& Sassone-Corsi, P. A direct role of SRY and SOX proteins in pre-mRNA splicing. Proc. Natl Acad. Sci. USA 99, 1146-1151 (2002)

\section{ONCOGENES}

\section{Dancing the two-step}

Regulation by protein degradation has become a recurring theme in cancer biology: several oncoproteins, including $\beta$-catenin, are controlled by being targeted for degradation. But biological control is seldom as simple as it seems. In EMBO Reports, Frank Staal and colleagues reveal that phosphorylation, not degradation, might be the key to regulating $\beta$-catenin's activity.

$\beta$-catenin leads a double life: it's a component of epithelial junctions but also a coactivator of the TCF family of transcription factors. Cell proliferation is stimulated by $\beta$-catenin-mediated induction of TCF's targets, and $\beta$ catenin levels are normally kept in check by a 'destruction complex' containing the APC tumour-suppressor protein and GSK3 $\beta$. The accepted view is that phosphorylation of $\beta$ catenin by GSK3 3 allows it to be recognized by a second complex that conjugates $\beta$-catenin with ubiquitin. The physiological switch that blocks $\beta$-catenin's destabilization is the extracellar signalling molecule Wnt, which indirectly prevents $\beta$-catenin from being phosphorylated by GSK3 $\beta$.

But is simply increasing the level of $\beta$-catenin sufficient to drive transcription of TCF-target genes? To find out, the authors blocked $\beta$-catenin degradation using a proteasome inhibitor and measured the transcription of TCF-target genes using a reporter construct. Surprisingly, stabilizing $\beta$ catenin did not increase transcription of the reporter gene.

The authors reasoned that dephosphorylation of $\beta$-catenin might be needed not just to protect it from destruction, but also to activate its function as a transcriptional coactivator. So they measured levels of dephosphorylated $\beta$-catenin under four different conditions, and found that dephosphorylation of $\beta$-catenin was indeed required for its activation.

Cath Brooksbank, Editor Nature Reviews Cancer

\section{0) References and links}

ORIGINAL RESEARCH PAPER Staal, F. J. T. et al. Wnt signals are transmitted through $\mathrm{N}$-terminally dephosphorylated $\beta$-catenin. EMBO Rep. 3, 63-68 (2002)

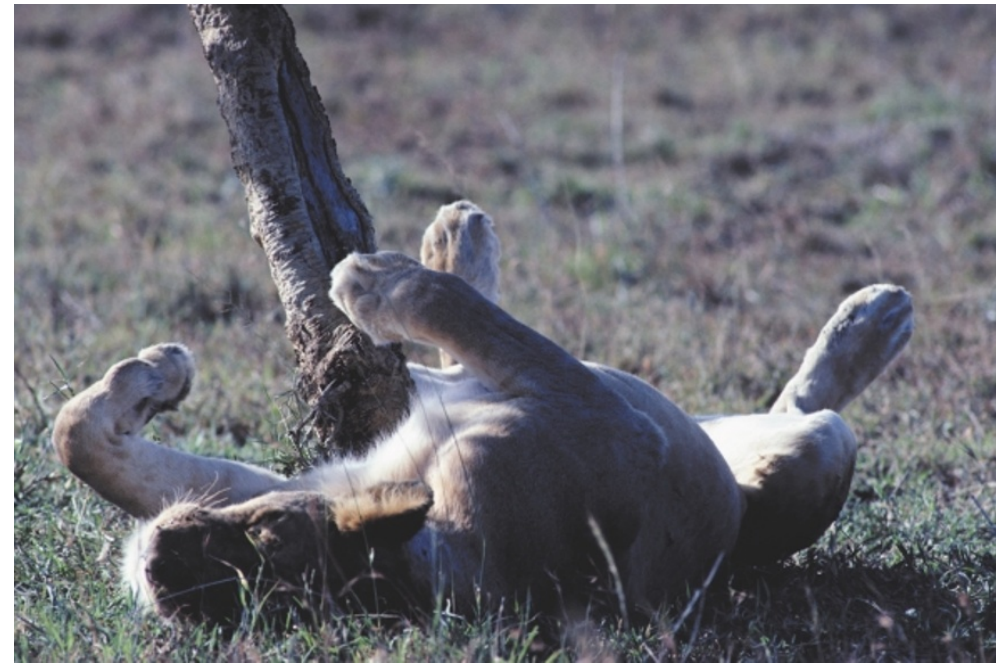

ENDOCRINOLOGY

\section{Time to relax}

The hormone relaxin favours the growth and remodelling of reproductive tissues during pregnancy. But despite the fact that relaxin was one of the first reproductive hormones to be identified, its receptor has remained undiscovered. Hsu and colleagues now report in Science that they have matched up the lonely hormone with the orphan receptors LGR7 and LGR8.

Although relaxin is structurally related to insulin, some data indicated that it might bind to a G-protein-coupled receptor (GPCR) instead of a tyrosine-kinase receptor. So, the authors tested whether any of the orphan leucine-rich GPCRs (LGRs) might be the missing relaxin receptor.

They expressed LGR7 or its relative LGR8 (which was discovered based on GenBank searches for genes with similar structure to glycoprotein hormone receptors) in cells and found that relaxin could indeed increase cyclic AMP levels in these cells in a dose-dependent manner, whereas it had no effect on cells that did not express these receptors. Crosslinking analysis showed that relaxin can bind to the extracellular domain of LGR7. Moreover, the soluble extracellular domain of LGR7 inhibited the effect of relaxin on cells. This inhibition was also observed in vivo, as pregnant mice treated with the extracellular domain of LGR7 had a delayed birth and reduced nipple size.

But the two receptors are expressed in many tissues, indicating that relaxin is far more versatile than just a pregnancy hormone. This is consistent with studies in humans, which have shown that, among other functions, relaxin is also important for cardiac function and for the formation of new blood vessels.

In rats, mice and other model organisms, relaxin functions during late pregnancy, when it promotes growth and dilation of the cervix, growth and quiescence of the uterus, and growth and development of the mammary gland and nipple. So, the authors suggest that the development of agonists and antagonists for its newly discovered receptors might help to solve problems related to preterm labour and delivery. In humans, however, as Richard Ivell points out in an accompanying Perspectives article, relaxin peaks during the first term of pregnancy, and is probably involved in implantation of the fetus. Whatever the exact function of relaxin, it is safe to say that the discovery of its receptors is bound to accelerate research on this versatile hormone.

Raluca Gagescu

6) References and links

ORIGINAL RESEARCH PAPER Hsu, S. Y. et al. Activation of orphan receptors by the hormone relaxin. Science $\mathbf{2 9 5}$, 671-674 (2002)

FURTHER READING Ivell, R. This hormone has been relaxin' too long. Science $\mathbf{2 9 5}$ $637-638$ (2002) 